\title{
Detección hematológica de Anaplasma phagocytophilum en caballos de la provincia de Chiclayo, Perú
}

\author{
Hematological detection of Anaplasma phagocytophilum in horses in the \\ province of Chiclayo, Peru
}

\author{
Deysi Masgo C. ${ }^{1}$, Luis Hoyos S. ${ }^{1,3}$, Olga Li E. ${ }^{1}$, Luis Gómez P. ${ }^{2}$, Leyla Ramires V. ${ }^{1}$, \\ Grecia Álvarez M. ${ }^{1}$, Miguel Cervantes S. ${ }^{1}$, Álvaro Vásquez Y. ${ }^{1}$
}

\section{Resumen}

El objetivo del estudio fue detectar Anaplasma phagocytophilum en caballos de la provincia de Chiclayo, Perú, utilizando técnicas hematológicas. Se colectaron 100 muestras de sangre de caballos aparentemente sanos y con antecedentes de exposición a garrapatas. El estudio hematológico consistió en la realización del hemograma y la evaluación de frotis sanguíneos teñidos con Wright y naranja de acridina para la búsqueda de cuerpos de inclusión y mórulas del agente patógeno. El 9\% de caballos (9/100) presentaron estructuras compatibles con cuerpos de inclusión o mórulas de A. phagocytophilum, por lo que fueron considerados como «positivos». Este grupo de animales presentó leves alteraciones en la serie leucocítica y trombocítica. Los hallazgos indican la presencia de $A$. phagocytophilum en caballos en el Perú.

Palabras clave: Anaplasma phagocytophilum; caballos; técnicas hematológicas

\section{Abstract}

The aim of this study was to detect Anaplasma phagocytophilum in horses from the province of Chiclayo, Peru, using haematological techniques. One hundred blood samples were collected from apparently healthy horses with a history of exposure to ticks. The haematological study consisted of blood count and the evaluation of blood smears stained with Wright and acridine orange to search for inclusion bodies and morulae of

\footnotetext{
${ }^{1}$ Laboratorio de Patología Clínica Veterinaria, Facultad de Medicina Veterinaria, Universidad Nacional Mayor de San Marcos, Lima, Perú

${ }^{2}$ Laboratorio de Epidemiología y Economía Veterinaria, Facultad de Medicina Veterinaria, Universidad Nacional Mayor de San Marcos, Lima, Perú

${ }^{3}$ E-mail: luis.hoyos@unmsm.edu.pe
}

Recibido: 13 de enero de 2019

Aceptado para publicación: 21 de octubre de 2019. 
the pathogen. Results showed that $9 \%$ of horses $(9 / 100)$ presented structures compatible with inclusion bodies or morulae of $A$. phagocytophilum, so they were considered as «positive». This group of animals presented slight alterations in the leukocytic and thrombocytic series. The findings indicate the presence of $A$. phagocytophilum in horses in Peru.

Key words: Anaplasma phagocytophilum; horses; hematological techniques

\section{INTRODUCCIÓN}

Anaplasma phagocytophilum es una bacteria gram negativa intracelular obligatoria que infecta granulocitos, principalmente neutrófilos, en cuyos citoplasmas forma una «microcolonia» denominada «mórula» (Woldehiwet, 2010). Es capaz de infectar a un gran número de mamíferos; sin embargo, la enfermedad clínica solo ha sido documentada en perros, ovejas, cabras, caballos, gatos y humanos, entre otros (Diniz y Breitschwerdt, 2011), denominándose anaplasmosis granulocítica.

La transmisión de la enfermedad se produce principalmente por la mordedura de una garrapata infectada del género Ixodes a un individuo susceptible (Pusterla y Madigan, 2014). La distribución de la enfermedad está asociada a la presencia de la garrapata vector, la cual se encuentra distribuida principalmente en el hemisferio norte. En el Perú ha sido reportada en el departamento de Amazonas (Macedo et al., 1994), y en el departamento de Piura (Glenny et al., 2004) infestando animales domésticos y silvestres.

Según el Centro para el Control y la Prevención de Enfermedades de los Estados Unidos (CDC), la Anaplasmosis Granulocítica Humana (AGH) es una enfermedad infecciosa de carácter zoonótico cuyos reportes se han ido incrementando anualmente. De 348 casos en 2000 a 4151 casos en 2016, con una tasa de letalidad estimada inferior al $1 \%$. Así mismo, tiene una presentación clínica inespecífica, siendo el síntoma más común la fiebre, seguido por dolor de cabeza, dolor muscular, malestar general, resfrío, náuseas, dolor abdominal y tos (CDC, 2018).

La Anaplasmosis Granulocítica Equina (AGE) fue reportada por primera vez en caballos al norte de California en 1960, habiéndose diseminado dentro de Estados Unidos, Canadá y Europa (Pusterla y Madigan, 2014) y en países de Sudamérica como Chile (Conejeros, 2012), Brasil (Farias et al., 2015) y Venezuela (Arraga et al., 1992; Castellanos et al., 2010). La presentación de la enfermedad está en función a la edad de los caballos, siendo más afectados los de mayor edad (Pusterla y Madigan, 2015); sin embargo, la enfermedad es autolimitante, habiéndose reportado caballos infectados asintomáticos (Madigan, 1993).

El diagnóstico se basa en la anamnesis, presentación de signos clínicos y evaluación hematológica en primera instancia. La técnica hematológica consiste en la realización del hemograma y la evaluación del frotis sanguíneo para la búsqueda de cuerpos de inclusión o mórulas en el citoplasma de neutrófilos y eosinófilos (Gribble, 1969). La mórula puede ser evidenciada entre menos del 1\% hasta en 20-30\% de los neutrófilos durante los primeros días de la infección (fase aguda) (Madigan y Pusterla, 2000); sin embargo existen reportes de la permanencia de la bacteria en circulación periférica en caballos meses después de su recuperación clínica. La sensibilidad y especificidad de la técnica es limitada, por ello es necesario el uso de técnicas 
confirmatorias como el PCR, técnica altamente sensible y específica; sin embargo, el Gold Standard es la prueba serológica de inmunofluorescencia indirecta (IFI) (CDC, 2018).

En el Perú, los caballos cumplen un rol cultural (Caballo Peruano de Paso), social (policía), deportivo (caballos de carrera), interactivo (caballos de paseo), e incluso terapéutico (equinoterapia), que hace estrecha su relación con el hombre favoreciendo la transmisión de enfermedades zoonóticas. La detección hematológica de $A$. phagocytophilum es, por lo tanto, de importancia para la salud pública. El diagnóstico temprano de la enfermedad es crucial para la aplicación de un correcto tratamiento y recuperación del paciente, ya que la mortalidad está altamente relacionada con el tiempo de evolución de la enfermedad, tanto en humanos como en caballos.

\section{Materiales y Métodos}

La colección de muestras se realizó en la provincia de Chiclayo, departamento de Lambayeque, ubicada en la costa norte del Perú, que presenta un clima subtropical con temperaturas promedio de $15.4-28.8^{\circ} \mathrm{C}$ (SENAMHI, 2019).

Se colectaron 100 muestras de sangre $(3 \mathrm{ml})$ en tubos comerciales con EDTA de caballos de cuatro haras en marzo de 2015. Para el muestreo se consideró la totalidad de animales de cada establecimiento, sin distinción de sexo, edad o raza. Los animales se encontraban aparentemente sanos el día de la toma de muestra, y todos tenían antecedentes de exposición a garrapatas.

El procesamiento de muestras para los recuentos hematológicos se llevó a cabo en el Centro Veterinario «Más que Patas» de la ciudad de Chiclayo, la evaluación de los frotis sanguíneos teñidos con Wright en el Labora- torio de Patología Clínica Veterinaria de la Facultad de Medicina Veterinaria (FMV) de la Universidad Nacional Mayor de San Marcos (UNMSM) y la evaluación de los frotis teñidos con naranja de acridina en el Laboratorio de Epidemiología y Economía Veterinaria de la FMV-UNMSM, estos últimos ubicados en la ciudad de Lima.

Se determinó la serie eritrocítica (hematocrito [\%], hemoglobina [g/dl], recuento total de glóbulos rojos $\left.\left[\mathrm{x} 10^{6} / \mu 1\right]\right)$, la serie leucocítica (recuento total de leucocitos $\left[\mathrm{x} 10^{3} / \mu 1\right]$, recuento diferencial relativo y absoluto), y la serie trombocítica (recuento total de plaquetas), de acuerdo a lo establecido por Benjamin (1991). Además, se observaron los frotices sanguíneos teñidos con Wright y naranja de acridina (Gribble, 1969) para la búsqueda de cuerpos de inclusión y mórulas (Uehlinger et al., 2011).

Para la visualización de los frotis sanguíneos teñidos con Wright se utilizó el microscopio de inmersión Leica DM 500 con objetivo de 100X para determinar el porcentaje de células circulantes con cuerpos de inclusión (CI) y mórulas y, a su vez, el número promedio de CI y mórulas/células. Este procedimiento se realizó identificando al menos 200 células (entre neutrófilos y eosinófilos) (Franzén et al., 2009) y se repitió 10 veces por cada frotis de sangre. La búsqueda de cuerpos de inclusión o mórulas se realizó tanto en la monocapa como en la cola de los frotis sanguíneos.

En el caso de CI y mórulas compatibles con A. phagocytophilum se utilizó la tinción naranja de acridina (Forbes, 2009). Los frotis fueron examinados en la monocapa y cola y por lo menos 200 células (entre neutrófilos y eosinófilos) fueron evaluadas, siguiendo el mismo procedimiento indicado para la tinción Wright, pero utilizando el microscopio de fluorescencia ZEISS Axioskop 40 con objetivos de 40x y 100x a una longitud de onda de excitación de 450-490 nm y de emisión de $515 \mathrm{~nm}$. 
Cuadro 1. Variables hematológicas de nueve caballos positivos a Anaplasma phagocytophilum, aparentemente sanos y con antecedentes de exposición a garrapatas, de la provincia de Chiclayo (Lambayeque, Perú). 2015

\begin{tabular}{|c|c|c|c|c|c|}
\hline & Variable & Media & D.E. & Rango & $\begin{array}{l}\text { Valores } \\
\text { referenciales }\end{array}$ \\
\hline \multirow{3}{*}{$\begin{array}{l}\text { Serie } \\
\text { eritrocítica }\end{array}$} & Eritrocitos $\left(\times 10^{6} / \mu \mathrm{l}\right)$ & 8.6 & 0.4 & $8.00-9.33$ & $8.2^{\mathrm{a}}-12.2^{\mathrm{a}}$ \\
\hline & Hemoglobina (g/dl) & 12.2 & 1.1 & $10.4-13.7$ & $13^{a}-17^{a}$ \\
\hline & Hematocrito (\%) & 35.0 & 3.5 & $30.0-40.0$ & $32^{\mathrm{a}}-48^{\mathrm{a}}$ \\
\hline \multirow{13}{*}{$\begin{array}{l}\text { Serie } \\
\text { leucocítica }\end{array}$} & Leucocitos $\left(\mathrm{x} 10^{3} / \mu \mathrm{l}\right)$ & 10.9 & 3.4 & $6.6-16.9$ & $8.00^{\mathrm{a}}-14.3^{\mathrm{a}}$ \\
\hline & Valores relativos & & & & \\
\hline & Neutrófilos (\%) & 59.0 & 9.7 & $44.0-77.0$ & $30-75^{\mathrm{b}}$ \\
\hline & Linfocitos (\%) & 39.0 & 9.8 & $21.0-49.0$ & $25-60^{\mathrm{b}}$ \\
\hline & Monocitos (\%) & 0 & 0.7 & $0-2$ & $1-8^{b}$ \\
\hline & Eosinófilos (\%) & 1.0 & 1.1 & $0-3$ & $1-10^{\mathrm{b}}$ \\
\hline & Basófilos (\%) & 0 & 0.5 & $0-1$ & $0-3^{b}$ \\
\hline & Valores absolutos & & & & \\
\hline & Neutrófilos $\left(\times 10^{3} / \mu \mathrm{l}\right)$ & 6.40 & 1.9 & $4.0-9.9$ & \\
\hline & Linfocitos $\left(\times 10^{3} / \mu \mathrm{l}\right)$ & 4.30 & 1.9 & $1.8-5.9$ & \\
\hline & Monocitos $\left(\mathrm{x} 10^{3} / \mu \mathrm{l}\right)$ & 0.03 & 0.1 & $0-0.2$ & \\
\hline & Eosinófilos $\left(\times 10^{3} / \mu \mathrm{l}\right)$ & 0.1 & 0.1 & $0-0.3$ & \\
\hline & Basófilos $\left(\times 10^{3} / \mu \mathrm{l}\right)$ & 0.03 & 0.05 & $0-0.1$ & \\
\hline $\begin{array}{l}\text { Serie } \\
\text { trombocítica }\end{array}$ & Plaquetas $\left(\mathrm{x} 10^{3} / \mu \mathrm{l}\right)$ & 183 & 56.3 & $132-276$ & $100^{\mathrm{a}}-500^{\mathrm{a}}$ \\
\hline
\end{tabular}

a Valores de referencia según Lording (2008); ${ }^{b}$ Valores de referencia según Jerez y Rudolph (1979)

\section{Resultados}

El 9\% de los caballos (9/100) evidenciaron estructuras compatibles con cuerpos de inclusión y mórulas de Anaplasma phagocytophilum en frotis de sangre periférica teñidos con Wright (Figura 1) y naranja de acridina (Figura 2), siendo considerados positivos al patógeno. Los resultados de las variables hematológicas de los nueve animales positivos, así como los valores referenciales se muestran en el Cuadro 1.
Cuadro 2. Distribución de caballos positivos a estructuras compatibles con Anaplasma phagocytophilum, según la presencia de trombocitopenia en caballos aparentemente sanos y con antecedentes de exposición a garrapatas (Chiclayo, Perú). 2015

\begin{tabular}{lcc}
\hline & $\begin{array}{c}\text { Con } \\
\text { trombocitopenia } \\
\left(<183 \times 10^{3} / \mu 1\right)\end{array}$ & $\begin{array}{c}\text { Sin } \\
\text { trombocitopenia } \\
\left(>183 \times 10^{3} / \mu 1\right)\end{array}$ \\
\hline $\begin{array}{l}\text { Caballos } \\
(\mathrm{n})\end{array}$ & 6 & 3 \\
$\begin{array}{l}\text { Plaquetas } \\
\left(\mathrm{x} 10^{3} / \mu 1\right)\end{array}$ & 148 & 252 \\
\hline
\end{tabular}



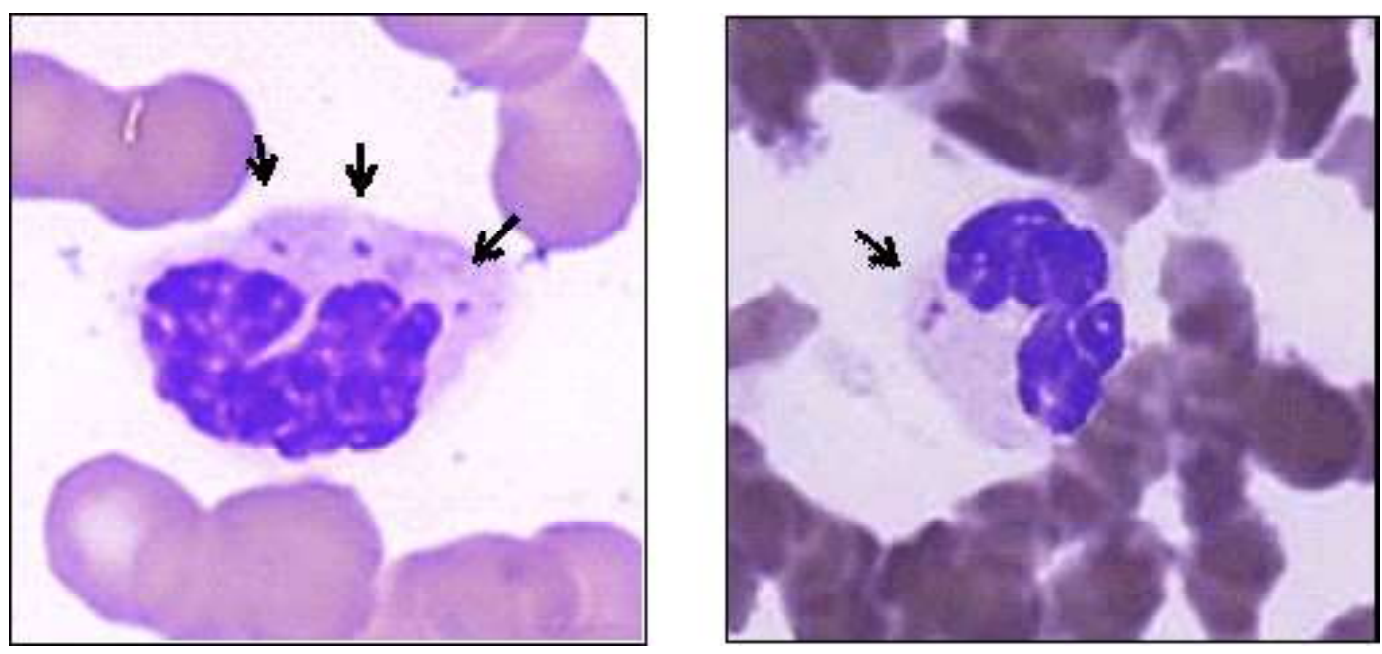

Figura 1. Frotis de sangre de caballo. Neutrófilos con presencia de estructuras en citoplasma (flechas) compatibles con cuerpos de inclusión de Anaplasma phagocytophilum. Tinción Wright. 100X

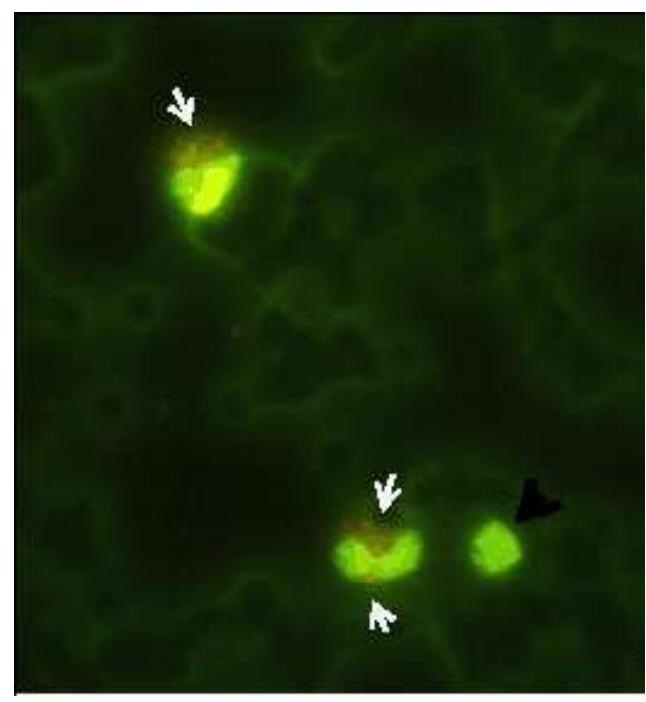

Figura 2. Frotis de sangre de caballo. Neutrófilos con evidencia de estructuras fluorescentes (naranja) (flechas blancas) en el citoplasma, compatibles con cuerpos de inclusión o mórulas de Anaplasma phagocytophilum. Se evidencia, además, un linfocito normal (flecha negra). Tinción Naranja de Acridina. 40X
El Cuadro 2, muestra la distribución de los animales «positivos» según la presencia o no de trombocitopenia, así como la media del conteo de plaquetas en cada grupo.

\section{Discusión}

La evaluación hematológica para la detección de Anaplasma phagocytophilum en caballos evidenció 9\% de animales con estructuras compatibles con cuerpos de inclusión o mórulas de Anaplasma phagocytophilum en el citoplasma de neutrófilos (Cuadro 1). Las estructuras visualizadas con la tinción Wright fueron cuerpos de inclusión, similares a las encontradas por Arraga et al. (1992), a los que denominó «cuerpos elementales o iniciales». Algo similar reportó Gribble (1969) quién observó inclusiones individuales a las que denominó «inclusiones tempranas pequeñas». Estas estructuras podrían corresponder a la fase de internalización de la bac- 
teria antes de la replicación (Troese y Carlyon, 2009). Sin embargo, las estructuras fluorescentes visualizadas con la tinción naranja de acridina corresponderían a mórulas, debido a su forma y distribución en el citoplasma de los neutrófilos.

Cabe mencionar que los animales evaluados se encontraban aparentemente sanos el día de la toma de muestra, lo cual coincide con lo referido por Castellanos et al. (2010) quienes reportaron el hallazgo de estructuras compatibles con A. phagocytophylum en el frotis sanguíneo de $32.9 \%$ de caballos asintomáticos provenientes de dos hatos del estado Apure, Venezuela.

La evaluación hematológica de los caballos «positivos» (Cuadro 2) mostraron leve leucocitosis, lo cual estuvo relacionada a un aumento en el valor absoluto de neutrófilos, lo cual podría darse como respuesta al estrés durante la toma de muestra (leucocitosis fisiológica). A su vez, se evidenció moderada linfopenia en algunos y linfocitosis en otros; hallazgos que concuerdan con Franzén et al. (2005).

Si bien la principal alteración hematológica de la infección con A. phagocytophilum es la trombocitopenia (Pusterla et al., 2000), solo el 56\% de los animales «positivos» presentaron trombocitopenia (6/9), lo cual difiere con otros estudios (Franzén et al., 2005, 2009) donde se evidenció trombocitopenias en todos los animales infectados. No hay reportes de alteraciones en el conteo de plaquetas en animales infectados asintómáticos; sin embargo, Franzén (2008) demostró la persistencia de la bacteria en la sangre detectada por PCR de sangre periférica hasta por tres meses después la recuperación clínica de caballos infectados experimentalmente.

En los animales «positivos» $(9 / 100) \mathrm{se}$ evidenciaron estructuras compatibles con cuerpos de inclusión o mórulas en un rango de 0.5 a $3 \%$ de neutrófilos (es decir, de 1 a 6 por cada 200 neutrófilos). Franzén et al. (2005) encontraron entre 0.5 y $16 \%$ de neutrófilos infectados, en tanto que Madigan y Pusterla (2000) refirieron que el porcentaje de neutrófilos con inclusiones varía en todo momento y entre caballos, presentándose entre menos de $1 \%$ y hasta en 20 y $30 \%$ de neutrófilos. Gribble (1969) evidenció el porcentaje más alto de infección (73\%) utilizando las tinciones giemsa y naranja de acridina.

Los animales en este estudio se encontraron aparentemente sanos el día de la toma de muestra, de allí que no cursaban la fase aguda de la enfermedad. Esto indica tres posibilidades: la primera, que los caballos hayan cursado con picos de fiebre por periodos cortos que pasaron desapercibidos por los criadores, coincidiendo con Franzén et al. (2005), quienes evidenciaron cuerpos de inclusión 2.6 días después del inicio de la fiebre, siendo por ello que el número de neutrófilos infectados fue bajo dado que el muestreo se realizó al inicio de la aparición de las inclusiones. La segunda es que haya un mecanismo de adaptación de los caballos de la zona con el agente, como lo ocurrido en áreas endémicas, donde la infección subclínica con $A$. phagocytophilum es común, y los caballos desarrollan anticuerpos contra este agente sin haber presentado signos clínicos (Madigan, 1993). Y la tercera posibilidad es que ocurra algo parecido a lo evidenciado en ovejas con infección persistente, las cuales tras la recuperación de la enfermedad clínica, no manifiestan signos clínicos, pero hay evidencia de la bacteria en sangre periférica (Stuen et al., 2001).

\section{Conclusiones}

- El presente estudio constituye el primer reporte de detección hematológica Anaplasma phagocytophilum en caballos en el Perú, lo que evidencia la exposición al agente zoonótico. Por lo tanto, la anaplasmosis granulocítica debería considerarse en la lista de diagnósticos 
diferenciales de enfermedades febriles, tanto en humanos como en caballos.

- La presentación de la enfermedad sería de tipo subclínico, dado que los animales se encontraron aparentemente sanos.

\section{Literatura Citada}

1. Arraga CM, Finol G, Parra $O$, Riquelme M, Savedra A. 1992. Ehrlichiosis equina en el estado Zulia, Venezuela. Reporte de 232 casos. Rev Cient-Fac Cien V 2: 41-52.

2. Benjamín M. 1991. Manual de patología clínica en veterinaria. México: Limusa. $421 \mathrm{p}$.

3. Castellanos R, Canelón J, Calzolaio V, Aguinaco F, López A, Montesinos R. 2010. Estudio Hematológico y detección de hemoparásitos en caballos criollos venezolanos de dos hatos del Estado de Apure, Venezuela. Rev Cient-Fac Cien V 20: 153-160.

4. [CDC] Centers for Disease Control and Prevention. 2018. Anaplasmosis. [Internet]. Disponible en: https:// www.cdc.gov/anaplasmosis/stats/ index.html

5. Conejeros C. 2012. Diagnóstico serológico de Anaplasma phagocytophilum en caballos Fina Sangre de Carrera perteneciente al Valparaíso Sporting Club Viña del Mar. Tesis de Médico Veterinario. Chile: Univ. Viña del Mar. 72 p.

6. Diniz P, Breitschwerdt E. 2011. Anaplasma phagocytophilum infection (Canine Granulocytotropic Anaplasmosis). En: Greene C (ed). Infectious diseases of the dog and cat. $4^{\text {th }}$ ed. Elsevier. p 227-238.

7. Farias M, Rodrigues FC, Soares FA, da Costa F. 2015. Serological evidence of exposure to Anaplasma phatocytophilum in horses from the Rio de Janeiro State mounted police bred in the urban zone. Cienc Anim Bras 16: 377-387. doi: 10.1590/1089-6891v16i319865
8. Forbes B. 2009. Principios generales de la microbiología clínica. En: Diagnóstico microbiológico. $12^{\circ}$ ed. Madrid: Panamericana. p 88-89.

9. Franzén P. 2008. On Anaplasma phagocytophilum in horses. $\mathrm{PhD}$ Thesis. Uppsala: Swedish University of Agricultutral Sciences. $67 \mathrm{p}$.

10. Franzén P, Aspan A, Egenvall A, Gunnarsson A, Karlsam E, Pringle J. 2009. Molecular evidence for persistence of Anaplasma phagocytophilum in the absence of clinical abnormalities in horses after recovery from acute experimental infection. J Vet Intern Med 23: 636-642. doi: 10.1111/ j.1939-1676.2009.0317.x

11. Glenny M, Mendoza L, Falconi R. 2004. Detección de anticuerpos contra Borrelia burgdorferi e identificación de garrapatas ixodidas en Piura y Amazonas, Perú. Rev Peru Med Exp Salud Publica 21: 23-27.

12. Gribble DH. 1969. Equine ehrlichiosis. J Am Vet Med Assoc 155: 462-469.

13. Jerez M, Rudolph W. 1979. Valores hematológicos, proteína plasmática total y fibrinógeno en equinos fina sangre de carrera en «training». Arch Med Vet 11: 72-75.

14. Lording PM. 2008. Erythrocytes. Vet Clin Pract 24: 225-237. doi: 10.1016/ j.cveq.2008.04.002

15. Macedo S, Cuadra AL, Arévalo J, Jove I. 1994. Borreliosis de Lyme: probables vectores implicados en la transmisión del agente etiológico. Rev Per Med Trop UNMSM 8: 27-32.

16. Madigan JE. 1993. Equine ehrlichiosis. Vet Clin N Am-Equine 9: 423-428. doi: 10.1016/S0749-0739(17)30408-X

17. Madigan J, Pusterla N. 2000. Ehrlichial diseases. Emerg Infect Dis 16: 487-499.

18. Pusterla N, Madigan J, Asnovich KM, Chae J, Derock E, Leutenegger CM, Berger J, et al. 2000. Experimental inoculation with human granulocytic ehrlichia agent derived from high- and 
low- passage cell culture in horses. J Clin Microbiol 38: 1276-1278.

19. Pusterla N, Madigan J. 2014. Anaplasma phagocytophilum infection. En: Sellon C, Long M (eds). Equine infectious diseases. $2^{\text {nd }}$ ed. China: Elsevier. p 344-347.

20. Pusterla N, Madigan J. 2015. Equine Granulocytic Anaplasmosis (formerly ehrlichiosis). In: Robinson N, Sprayberry $\mathrm{K}$ (eds). Robinson's current therapy in equine medicine. $7^{\text {th }}$ ed. USA: Elsevier. p 193-195.

21. SENAMHI] Servicio Nacional de Meteorología e Hidrología del Perú. 2019. Información de tiempo y clima. [Internet], [13 enero 2019]. Disponible en: https://www.senamhi.gob.pe/

22. Troese MJ, Carlyon JA. 2009. Anaplasma phagocytophilum dense-- cored organisms mediate cellular adherence through recognition of human $\mathrm{P}$ selectin glycoprotein ligand 1 . Infect Immun 77: 4018-4027. doi: 10.1128/ IAI.00527-09

23. Stuen S, Djuve R, Bergstrom K. 2001. Persistence of granulocytic ehrlichia infection during wintertime in two sheep flocks in Norway. Acta Vet Scand 42: 347-353. doi: 10.1186/1751-0147-42-347

24. Uehlinger FD, Clancey NP, Lofstedt J. 2011. Granulocytic anaplasmosis in a horse from Nova Scotia caused by infection with Anaplasma phagocytophilum. Can Vet J 52: 537-540.

25. Woldehiwet $Z$. 2010. The natural history of Anaplasma phagocytophilum. Vet Parasitol 167: 108-122. doi: 10.1016/ j.vetpar.2009.09.013 